\title{
Analyse Des Situations Problématiques De Quelques Manuels Scolaires En Rapport Avec l'Ecologie Et l'Education Relative À l'Environnement Dans Les Programmes Des Sections Internationales Du Baccalauréat Marocain, SVT-Option Français
}

\author{
Bouchra Bekhat, \\ Mourad Madrane, \\ Rachid Janati-Idrissi, \\ Rajae Zerhane, \\ Mohamed Laafou,
}

Laboratoire Interdisciplinaire de Recherche en Ingénierie Pédagogique École Normale Supérieure, Université Abdelmalek Essaadi, Tétouan, Maroc

Doi:10.19044/esj.2020.v16n15p127 URL:http://dx.doi.org/10.19044/esj.2020.v16n15p127

\section{Résumé}

Désormais, les sociétés seront confrontées à des problèmes de développement, de surpopulation et d'environnement qui impliqueraient de la part du citoyen des compétences et des prédispositions aptes à permettre une approche critique de ces problèmes. L'école et les actions éducatives seraient les seuls garants d'une éducation à l'environnement susceptibles de développer des valeurs et des habilités permettant le développement de comportements favorables à la protection et à la préservation des milieux naturels. Cependant, le constat général montre un écart important entre les objectifs visés et la qualité des actions éducatives réellement déployées. Pour ses actions, le travail mené est relatif à la nature et à l'évaluation de la pertinence didactique des textes introductifs ou situations problématiques proposés par différents manuels en rapport avec des thématiques écologiques et environnementales. Il vise, de ce fait, une analyse critique de ces textes, et ce pour décortiquer leurs contenus et leur pertinence didactique relativement aux objectifs visés de problématisation et d'enseignement. Aussi la méthodologie adoptée est-elle fondée principalement sur l'analyse des contenus. Les résultats obtenus aboutissent à la conclusion selon laquelle ces situations problèmes et ces textes introductifs ne peuvent pas constituer des documents didactiques pertinents pour motiver et mettre en œuvre des actions éducatives efficaces en rapport avec les objectifs de l'éducation relative à l'environnement. 
Mots-Clés : Education Relative À L'environnement, Action Éducative, Évaluation, Situation Problématique, Problématisation, Manuels Scolaires, Maroc

\title{
Analysis of the Problematic Situation of Some School Manuals in Relation to Ecology and Environmental Education in the Programs of the International Sections of the Moroccan Baccalaureate, SVT, Option-French
}

\author{
Bouchra Bekhat, \\ Mourad Madrane, \\ Rachid Janati-Idrissi, \\ Rajae Zerhane, \\ Mohamed Laafou,
}

Laboratoire Interdisciplinaire de Recherche en Ingénierie Pédagogique École Normale Supérieure, Université Abdelmalek Essaadi, Tétouan, Maroc

\begin{abstract}
Societies are faced with development, overpopulation, and environmental problems which imply on the part of the citizen certain competences and predispositions. This has led to a critical approach of these problems. School and educational actions would be the only guarantors of an environmental education which is likely to develop values and skills that allow the development of favourable behaviours for the protection and the preservation of the natural environments. The general observation shows an important difference between the objectives and the quality of the educational actions deployed. This paper focuses on a critical analysis of these texts in order to dissect their content and their didactic relevance to the objectives of teaching. The methodology adopted is based mainly on content analysis. The results obtained lead to the conclusion that such situations and introductory texts cannot constitute relevant didactic documents to motivate and implement effective educational actions which are related to the objectives of environmental education.
\end{abstract}

Keywords: Environnemental Education, Action, Evaluation, Problem Situation, Problematization, School Textbooks, Morocco 


\section{Introduction}

Dans un contexte régional et mondial marqué par des problématiques et des enjeux importants liés à l'environnement, le futur citoyen est appelé à comprendre, à être sensibilisé et à agir en faveur de l'environnement en adoptant des comportements idoines. Dans ce sens, il est appelé, parfois, à participer aux mécanismes de prise de décision et à inciter autrui à développer des attitudes adéquates.

En effet, ceci n'est possible que s'il est apte à comprendre et à analyser les phénomènes et les problématiques qui structurent et caractérisent son milieu ambiant. Le citoyen serait capable de comprendre et d'analyser ce milieu s'il est doté de compétences dont 1'acquisition se fait essentiellement dans le cadre scolaire.

En outre, des actions menées par le biais des médias ou des associations pourraient avoir un impact certain sur les valeurs et les attitudes des citoyens en relation avec l'environnement.

Cependant, l'école et les actions éducatives seraient les principaux garants d'une éducation à l'environnement apte à développer des valeurs, des habilités et des comportements favorables à la protection et à la préservation des milieux naturels. Dans ce sens, la finalité d'une éducation à l'environnement serait le développement d'une conception d'un monde plus viable, et ce à travers le développement d'une culture environnementale.

Des actions éducatives efficaces visant à atteindre cette finalité impliqueraient l'intervention d'enseignants compétents, capables de traduire des intentions éducatives en actions pédagogiques concrètes et conséquentes. Des constats relatifs aux comportements des apprenants vis-à-vis de l'environnement témoignent de l'existence d'un écart important entre les objectifs d'enseignement affichés et les connaissances et les capacités développées réellement. Ceci est valable pour l'enseignement en général et davantage pour l'éducation à l'environnement où il est question de développer des valeurs et de susciter des comportements éco-favorables.

\section{1- Problématique}

Depuis quelques années, le Maroc est confronté à la problématique de la qualité de l'enseignement en général et de l'enseignement des sciences en particulier. Des études et des évaluations nationales et internationales convergent vers l'idée d'un enseignement des sciences en échec, relativement aux compétences visées. Ainsi, selon Melhaoui et al. (2004), les taux d'échec et d'abandon d'étudiants dans les universités marocaines sont élevés, seul 10 $\%$ des étudiants d'une promotion obtiennent leur licence en quatre ans.

En ce qui concerne l'éducation à l'environnement, le constat est le même et il y aurait un écart important entre les objectifs visés et la qualité des actions éducatives réellement déployées. L'échec en question s'explique par 
la portée très limitée des actions éducatives en termesde connaissances stabilisées, de compétences et comportements développés. En ce qui concerne l'éducation à l'environnement, les actions développées auraient peu de retombées sur les connaissances et les comportements visés. De plus, les connaissances véhiculées ne seraient pas intégrées dans des comportements éco-favorables visibles et palpables.

Pour permettre un certain apprentissage et pour donner du sens aux apprentissages et montrer à l'élève à quoi sert tout ce qu'il apprend à l'école. Pour cela il est nécessaire de dépasser des listes de contenus et des descriptions proposées de manière magistrale et chercher comment enseigner autrement en utilisant des procédés didactiques axés sur le la problématisation des thématiques, situations et phénomènes à l'étude. Dans cette perspective, l'approche par compétences permettrait de placer les apprentissages par rapport à des situations qui ont du sens pour lui et à utiliser ses acquis dans ces situations. Adopter une méthode pédagogique se basant sur la démarche de résolution de problèmes serait un procédé important pour garantir une certaine motivation et pour impliquer l'apprenant mentalement. Un apprentissage implique la motivation, l'engagement et l'incitation à anticiper les choses. Ces aspects sont atteints si l'enseignant procède par problématisation et résolution de problème. Il n'en demeure pas moins qu'il ne s'agit pas d'une résolution d'un problème mathématique, mais un problème souvent rencontré dans les sciences de la vie et de la terre. La conception d'apprentissage ainsi véhiculée appartient aux courants constructivistes, socioconstructivistes qui sont en étroite relation avec le courant cognitiviste. Elle consiste à un processus d'acquisition de connaissances qui prend en considération l'activité de l'apprenant. Celui-ci devient le responsable de la construction de ses connaissances et acteur dans son apprentissage, contrairement aux approches transmissives. Le problème et la problématisation sont au cœur de l'approche par compétences Lage (2007).

Vouloir évaluer l'impact de cette éducation relative à l'environnement consiste tout d'abord à apprécier la qualité des actions éducatives entreprises. Il est à noter que ces actions éducatives s'inscrivent dans un système d'enseignement plus large qui comprend les programmes et manuels scolaires, la formation à l'enseignement, l'encadrement pédagogique, l'évaluation, le cadre institutionnel...etc. Par conséquent, la qualité des actions déployées est tributaire du fonctionnement global de ce système. Et vouloir approcher ce problème de qualité requiert une analyse de l'ensemble du système d'enseignement duquel ces actions émanent.

La problématique, en l'occurrence, est relative à la nature et à la pertinence didactique de situations introductives proposées par différents manuels scolaires en rapport avec des thématiques écologiques et environnementales en vigueur dans le système éducatif marocain. Il y a lieu 
de remarquer que ces modèles de situation sont largement partagés et utilisés par les enseignants. Pour les raisons invoquées, la problématique à l'étude revoie à la portée et aux implications didactiques possibles de ces textes introductifs. De ce fait, il est question d'analyser ces textes pour en décortiquer les contenus et les pertinences didactiques relativement aux objectifs visés de problématisation et d'enseignement.

\section{2- Cadre théorique}

\section{1- Concept d'éducation relative à l'environnement}

Depuis les années soixante, l'éducation à l'environnement s'est développée comme une forme d'éducation essentielle vers l'acquisition de valeurs pour la sensibilisation, la protection et le respect du patrimoine naturel. Depuis, l'éducation à l'environnement a évolué considérablement, passant d'une éducation en rapport avec la problématique de "nature» vers une éducation en lien avec la problématique de «l'environnement». Elle ne vise plus, désormais, simplement la sensibilisation, la protection et le respect de l'environnement mais elle devient partie intégrante des programmes éducatifs incluant des compétences transversales dont les finalités sont l'éducation à la paix, au développement durable et à la citoyenneté.

Qu'il s'agisse d'une éducation pour l'environnement, par l'environnement, relative à l'environnement, cette éducation vise à développer une conscience environnementale relative à la fragilité et à la complexité de notre environnement. Et le but ultime souhaité à travers cette éducation est l'incitation à l'action locale ou régionale favorable à la pérennité de la vie et de l'environnement global. Dans ce sens, la définition officielle de cette tendance pédagogique proposée durant la conférence de Tbilissi en 1977 met en valeur l'importance des connaissances, des valeurs, des comportements et des compétences pratiques nécessaires à des actions éco-favorables éventuelles. L'Agenda 21 élaborée au cours du Sommet mondial de la Terre de Rio en 1992 consacre un chapitre à l'éducation à l'environnement en mettant l'accent sur le développement durable et son importance pour le développement d'une culture de l'éco-citoyenneté. La Conférence de Bonn en 2009 considère de sa part l'éducation pour un développement durable comme étant le principal vecteur d'évolution des sociétés vers un mode de développement global (UNESCO, 2009).

\section{2 - Qu'est-ce qu'un savoir scientifique ?}

A la question qu'est-ce qu'un savoir scientifique, plusieurs réponses sont possibles selon les auteurs et les personnes interrogées (enseignants ou formateurs). Ceci est d'autant plus vrai si ces derniers n'ont pas reçu de formation en histoire et en épistémologie des sciences. C'est d'ailleurs le cas de la majorité des enseignants. Un enseignant sensé développer une culture 
scientifique devrait être conscient tout d'abord qu'un savoir scientifique n'est pas seulement un savoir qui permet de résoudre des problèmes ou de décrire des réalités ou des phénomènes ; c'est aussi un savoir qui permet de formuler de nouvelles questions qui relancent le processus de développement des connaissances. Cette caractéristique peut être rapprochée de l'idée de connaissance questionnant et questionnée de Bachelard (1972, p. 27). Cette dynamique conduit Popper (1985, pp 329-330) «à une représentation de la science comme processus ayant pour point de départ et pour terme la formulation de problèmes toujours plus fondamentaux et dont la fécondité ne cesse de s'accroître, en donnant le jour à d'autres problèmes encore inédits ». Pour sa part, Bachelard (1938) note que «pour un esprit scientifique, toute connaissance est une réponse à une question»; Popper affirme que la science commence par des problèmes (1991, p. 287; 1985, pp. 230, 329).Toutefois, un problème n'est pas seulement présent au début et à la fin d'un processus de construction de connaissance : «Car un problème est une difficulté, et comprendre un problème consiste à découvrir qu'il y a une difficulté et où gît la difficulté. Ce qui ne peut se faire qu'en découvrant pourquoi certaines solutions de prime abord ne marchent pas. » (Popper, 1991, p. 282).

Selon Orange $(2002,2005,2007)$, la nature des savoirs scientifiques fondamentaux serait marquée par les traits épistémologiques suivants :

- Les savoirs scientifiques fondamentaux sont relatifs à des problèmes d'explication et de caractérisation de phénomènes et de faits ;

- Ces savoirs scientifiques articulent au moins deux registres qui sont le registre empirique (faits et phénomènes à expliquer) et le registre des modèles (où sont développées des explications);

- Les savoirs scientifiques sont apodictiques, c'est-à-dire que ces savoirs ne se limitent à des descriptions (des faits, des phénomènes) ou des descriptions de fonctionnement, mais se rapportent plus aux conditions qui imposent l'adoption de certaines explications (conditions de possibilité) et qui sont en fait des nécessités sur des modèles (délimitation du champ des possibles par argumentations critiques) qui imposent la construction et la sélection de certaines explications et le rejet de certaines explications jugées impossibles.

\section{3- Qu'est-ce que problématisation ?}

Dewey et Bachelard (1993) s'accordent sur cinq caractéristiques de la problématisation :

1) elle renvoie à une activité complexe impliquant position, construction et résolution de problèmes ;

2) c'est une tentative de recherche de l'inconnu à partir du connu, c'està-dire l'identification de points d'appui à partir desquels interroger ;

3) c'est une dialectique de faits et d'idées, d'expériences et de théories ; 
4) c'est une pensée cadrée par des normes (intellectuelles, éthiques, techniques, pragmatiques, etc.) ;

5) c'est une schématisation fonctionnelle du réel qui renonce à tout embrasser et à reproduire la réalité mais vise plutôt à construire des outils pour penser et agir (Fabre, 2005b).

Selon Orange (2002), la problématisation n'est pas une compétence en rupture avec les savoirs en jeu. Le lien entre savoirs scientifiques et problématisation y est fort, ceux-ci ne pouvant pas être simplement considérés comme des solutions à des problèmes, mais comme la trace même de la problématisation. Ainsi, une démarche de problématisation est souhaitable voire nécessaire pour tout impliquer dans une entreprise d'enseignementapprentissage. Selon le cadre théorique de Christian Orange (2002), toute situation problématique peut être ramenée à un modèle marqué par un registre empirique et un registre de modèles (ou registre des explications). Il faut préciser que le modèle de Christian Orange est inspiré principalement des idées issues de l'épistémologie de Bachelard (1949) et Canguilhem (1988).

Il faut souligner que la problématisation n'est plus au service de la résolution du problème; la relation s'inverse : les hypothèses possibles servent la problématisation. Celle-ci se déploie en une seconde dimension, celle d'une problématisation explicite et mise en texte.

La position choisie consiste à considérer le problème comme le moyen d'accès au savoir scientifique (Orange, 2000) et non seulement comme point de départ d'investigations par les élèves. Il s'agit de les aider à construire des problèmes leur permettant d'accéder à un savoir raisonné et argumenté.

\section{4 - Les références épistémologiques et didactiques du concept de problème}

Un nombre important d'épistémologues accordent une place et une fonction centrale au problème dans l'activité et le processus de production du savoir scientifique. Bachelard (1938) note ainsi que« pour un esprit scientifique, toute connaissance est une réponse à une question ». Pour Karl Popper (1985, pp. 230, 329; 1991, p. 287), une activité scientifique débute par un ou des problèmes. Ces points de vue épistémologiques revoient à une conception rationaliste des sciences et de l'activité scientifique.

Dans une perspective scolaire, c'est une manière d'établir une rupture avec une conception empirico-positiviste largement partagée et véhiculée par un enseignement porteur de cette vision. Sur le plan didactique, plusieurs études sur le raisonnement concluent que les apprenants développent des stratégies spécifiques pour l'appropriation de compétences d'évaluation et d'argumentation faceàdes problèmes complexes (Pauen \& Wilkening, 1997; Zimmerman, 2000; Kuhn, 2001). 
Dans cette perspective, des études font état de l'argumentation proposée par Simonneaux $(2001 ; 2002)$ ou demande de prise de décision face à des sujets controversés (socio-scientific-issues) (Kolstø, 2001; Sadler et al., 2004). Des controverses telles que des situations problématiques relatives à l'environnement. Il faut souligner que l'analyse et la résolution des problèmes peuvent constituer des moments intéressants pour l'exercice d'une pensée réflexive par les apprenants. D'ailleurs, plusieurs auteurs s'accordent sur l'importance de la réflexivité dans les processus d'apprentissage. Une telle réflexivité développe la pensée réflexive qui se distingue des autres formes d'opérations cognitives parce qu'elle projette l'apprenant dans des moments de doute, d'hésitation, de difficulté mentale qui favorisent amplement le développement de cette pensée. Dans ce sens, Dewey (1933, p. 12), parle d' « une action de recherche, d'investigation, afin de se doter d'instruments de résolution du doute, de se stabiliser et de se débarrasser de l'état d'incertitude» Un enseignement fondé sur les problèmes rompt ainsi avec la tradition d'enseignement des sciences (Aikenhead, 2003). Face à la science-savoir des manuels, il y a les problèmes liés à la science-ignorance des laboratoires. En effet, pour Berlan (2002), ces problèmes correspondent à des ignorances età des interrogations. En fait, il s'agit d'adopter un cadre théorique qui est celui de l'apprentissage par problématisation fondé et inspiré des idées de Bachelard et de Canguilhem (1972).

\section{5- Situation problème}

Un problème correspond à une situation d'où il est possible de dégager des données. Une situation problème représenterait une énigme réelle à résoudre Astolfï (1993). Elle devrait permettre aux élèves de formuler des hypothèses et pour laquelle les apprenants se sentent démunis pour qu'ils investissent leurs connaissances antérieures et leurs idées et représentations. L'aboutissement de ce parcours mental débouche sur un questionnement qui mène à la proposition de nouvelles idées. Fabre (2003) semble pourtant suggérer qu'il est impossible de construire un problème avant de l'avoir résolu. Dans la présente étude, les situations problèmes sont considérées selon la caractérisation qu'en a proposée Sternberg (1999). L'élève est confronté à des situations pour lesquelles il ne possède aucune solution immédiate dans un répertoire prédéfini. Il lui faut alors reconnaître le problème, le définir, se l'approprier, proposer un raisonnement, formuler des stratégies de résolution, faire appel à ses ressources cognitives et établir des mécanismes de vérification et d'évaluation de la solution (ou des solutions) proposée (s).

Selon le modèle élaboré par Orange (2002) et le modèle de Fabre (2009), il y a une grille comportant six dimensions qui ont été retenues en fonction des objectifs d'analyse et d'évaluation de ces situations introductives ou problématiques. 
Six dimensions constitueraient les traits majeurs du processus de problématisation (Tableau 1). Une première dimension selon laquelle la problématisation peut être décrite comme étant une relation possible entre un problème et une solution. Une seconde dimension selon laquelle un travail de problématisation scientifique est activé si le texte propose des questions et des remarques permettant de sélectionner et de modéliser les faits pertinents par rapport aux conditions du problème. Une troisième dimension qui renvoie au critère de l'existence ou non de questions et de remarques proposés par le texte et permettant la reformulation et l'appropriation du problème lors de la problématisation. Une quatrième dimension relative à l'existence ou non dans le texte de questions et remarques permettant de recentrer le questionnement développé par l'apprenant lors de la problématisation. Une cinquième dimension relative aux remarques et questions proposés par le texte permettant de percevoir la relation entre problèmes et savoirs, d'une part, de faciliter l'articulation entre registre des faits empiriques (ou faits scientifiques établis) et le registre des modèles et la production d'hypothèses à travers des raisonnements ou des débats, d'autre part. Une sixième dimension en relation avec la teneur du texte en termes de questions et de remarques permettant la verbalisation et de mise en texte permettant la formalisation et la conceptualisation.

Selon cet auteur, une activité scientifique vise principalement l'élaboration de modèles explicatifs proposant des relations entre cause et effet qui renvoient en fait à des nécessités qui ne sont pas fondées simplement sur des constats empiriques mais principalement sur une activité de construction critique de modèles explicatifs. Ce qui confère à ces modèles leurs caractères scientifiques et conceptuels.

Tableau 1. Grille inspirée du cadre théorique exploitée par Christian Orange $(2005,2007)$

\begin{tabular}{|l|l|}
\hline Dimension ou trait 1 (Elément 1) & $\begin{array}{l}\text { - Une problématisation est une manière de penser la relation } \\
\text { problème solution ; }\end{array}$ \\
\hline Dimension ou trait 2 (Elément 2) & $\begin{array}{l}\text {-Texte comportant des questions et des remarques } \\
\text { permettant de sélectionner et de modéliser des faits selon } \\
\text { les conditions du problème ; }\end{array}$ \\
\hline Dimension ou trait 3 (Elément 3) & $\begin{array}{l}\text { - des questions et des remarques permettant d'inciter à la } \\
\text { reformulation du problème lors de la problématisation; }\end{array}$ \\
\hline Dimension ou trait 4 (Elément 4) & $\begin{array}{l}\text { - Des questions et des remarques permettant de recentrer le } \\
\text { questionnement de l'apprenant lors de la problématisation ; }\end{array}$ \\
\hline Dimension ou trait 5 (Elément 5) & $\begin{array}{l}\text { - Un travail scientifique caractérisé par une relation } \\
\text { dynamique entre savoirs et problèmes ; }\end{array}$ \\
& $\begin{array}{l}\text { - il vise principalement l'élaboration de modèles explicatifs } \\
\text { proposant des relations entre cause et effet qui renvoient en } \\
\text { fait à des nécessités qui ne sont pas fondées simplement sur } \\
\text { des constats empiriques mais principalement sur une } \\
\text { activité de construction critique de modèles explicatifs. } \\
\text { - Une problématisation articule deux registres : un registre } \\
\text { empirique (observation ou expérience commune) et un }\end{array}$ \\
\hline
\end{tabular}




\begin{tabular}{|l|l|}
\hline & $\begin{array}{l}\text { registre de modèles (ce qui renvoie à des explications qui } \\
\text { ne résultent pas d'un simple constat ou expériences } \\
\text { communes mais renvoient plutôt à des nécessités). (Phases } \\
\text { des hypothèses et des débats en classe) }\end{array}$ \\
\hline Dimension ou trait 6 (Elément 6) & $\begin{array}{l}\text { Le sixième élément de ce modèle renvoie au travail } \\
\text { langagier important dans toute activité scientifique ou } \\
\text { travail de conceptualisation (verbalisation ou élaboration } \\
\text { d'un texte écrit ou production d'un texte oral ou écrit). }\end{array}$ \\
\hline
\end{tabular}

Ce tableau est inspiré du cadre théorique de l'apprentissage par problématisation des sciences de la vie et de la terre développé par Orange (2007) et qui précise que ce cadre renvoie à des positions épistémologiques (Orange, 2002; 2005). Il faut souligner dans ce sens que selon Fabre (1993; 1999), ce cadre théorique s'inscrit dans une réflexion plus large sur la problématisation et ses liens avec les savoirs scientifiques et les apprentissages.

C'est ce travail de la pensée scientifique (toujours au sens large) qui confère des caractéristiques spécifiques aux problématisations développées : il ne s'agit pas simplement de construire un problème pour produire une solution, mais d'explorer et de «cartographier» le champ des possibles. Cette exploration a pour résultat un caractère essentiel des savoirs scientifiques.

Le cadre théorique en général et le modèle de Christian Orange (2002) en particulier ont servi de base pour élaborer une grille visant une analyse critique de quelques situations problématiques proposées par des manuels scolaires en vigueur dans le système d'enseignement marocain. Les manuels scolaires en question sont programmés dans la filière dite Baccalauréat international ainsi que dans le Tronc Commun. Il faut souligner que cette analyse va permettre encore d'évaluer la réforme du système d'enseignement, en particulier celle relative au changement des programmes et la langue d'enseignement des sciences de la vie et la terre.

\section{3- Méthodologie}

Ce travail de recherche se limite à l'analyse des manuels scolaires, plus précisément les parties des manuels dédiées à la présentation de textes introductifs qui constituent en fait des situations problématiques. En fait, le choix s'est porté sur quelques textes introductifs de chapitres de quelques manuels des sciences de la vie et de la terre Section Internationale du Baccalauréat Marocain (option Français) en rapport avec l'écologie et l'éducation relative à l'environnement pour les soumettre à une analyse fine eu égard le cadre théorique et les grilles d'analyse.

Le choix de ces manuels scolaires est dicté par le fait qu'ils sont récents et censés être utilisés par une élite d'apprenants marocains (du baccalauréat international). Il est à souligner que ces manuels s'inscrivent dans la 
perspective d'une réforme en relation avec la langue d'enseignement des sciences de la vie et de la terre et les manières de les enseigner. Les apprenants de ces filières sont sélectionnés sur la base des notes et du niveau linguistique en français. Le choix de ces manuels est justifié encore par les recommandations de la charte nationale de l'enseignement oùil est question de dispenser un enseignement de qualité des langues, de diversifier les langues d'enseignement et d'assurer une offre en termes de choix des langues d'enseignement.

Pour toutes les raisons invoquées et par rapport aux objectifs de recherche, l'outil méthodologique requis dans cette étude est la technique de l'analyse du contenu telle qu'elle a été théorisée et opérationnalisée par Bardin (1977).

L'analyse du contenu consiste en un ensemble d'instruments méthodologiques s'appliquant à des «discours» extrêmement diversifiés et fondés sur la déduction ainsi que l'inférence. Il s'agit, en fait, d'un travail d'interprétation qui se balance entre deux positions, d'une part, la rigueur de l'objectivité, et, d'autre part, la fécondité de la subjectivité (Bardin, 1977). L'analyse du contenu s'organise autour de trois phases chronologiques : la pré-analyse, l'exploitation du matériel (corpus de données et de faits) ainsi que le traitement des résultats, l'inférence et l'interprétation. Le choix des objets et du corpus ainsi que le travail d'analyse ont été fondés sur le cadre théorique et le cadre de Christian Orange (2002). En fait, l'analyse a été menée à travers une grille d'analyse à la base des idées développées par Orange. Ce cadre d'interprétation a servi de référence pour pouvoir faire des inférences et des interprétations des idées et du corpus en général contenus dans les différentes situations introductives ou problématiques proposées dans des chapitres de quelques manuels des sciences de la vie et de la terre, Section Internationale du Baccalauréat Marocain (option Français) qui renvoient à l'écologie et/ou l'éducation à l'environnement . Il est à noter que malgré le recours à une grille et un cadre de référence, inspiré de Bachelard et Canguilhem (1972), une part de subjectivité serait inhérente à tout travail d'inférence et d'interprétation.

\section{4- Résultats}

Le travail d'analyse mené a porté sur des situations problématiques de différents manuels scolaires, relativement aux thématiques environnementale et écologique dans le programme marocain.

4.1 -Situation 1 : (livre : SVT+ Plus tronc commun scientifique ; Chapitre 5 ; Les équilibres naturels ; $p: 73$, Editions Maarif)

\subsection{1-Texte relatif à la problématique du chapitre 5}


En aménageant le territoire, l'homme modifie son environnement naturel : il détruit parfois des milieux de vie. Cependant, il prend peu à peu conscience des dangers qu'il fait courir à la planète et commence à restaurer des milieux qu'il a transformés.

$\checkmark$ Comment l'homme a - t-il modifié et altéré son environnement?

$\checkmark$ Quels moyens met-il en œuvre pour préserver l'équilibre naturel ?

\subsection{2- Analyse de la situation 1}

- Le texte proposé ne comporte pas de faits empiriques ou faits scientifiquement établis qui puissent permettre la production d'hypothèses par l'apprenant ;

A la lecture du texte, apparaît la synthèse d'une leçon dans laquelle il y a proposition de certitudes et de propositions vraies. Une telle présentation empêche tout travail d'imagination ou de conception d'hypothèses explicatives sur les faits ou les phénomènes évoqués. De même, le contenu et la structuration du texte ne permettraient pas l'engagement de l'apprenant dans un travail d'investigation et de raisonnement scientifique ;

- $\quad$ Les idées de modification de l'environnement et de restauration des milieux ne doivent pas figurer dans le texte, mais doivent être proposées par les apprenants sous formes d'hypothèses. Cette activité qui consiste à proposer des idées-hypothèses est nécessaire dans toute activité de problématisation. En effet, ces propositions renvoient au registre des modèles et des explications et qui est fondamentale pour problématiser ou modéliser la solution du problème ;

La problématique proposée ne serait pas adéquate pour introduire le concept d'équilibre naturel. En effet, le document doit fournir des faits et des idées qui puissent permettre à l'apprenant de déduire l'idée d'équilibre naturel à partir d'une évolution d'un espace-temps naturel actuellement en équilibre ou d'un exemple d'une action négative provoquant la disparition progressive d'un équilibre naturel. Le texte proposé ne renvoie à aucun cas de figure mentionné.

Les idées de transformation, de modification, de destruction, de restauration, de préservation et d'altération ne doivent pas figurées dans le texte ou les questions car elles serviront ou constitueront des termes clés dans la formulation des hypothèses que l'apprenant doit développer. En conséquence, la divulgation de ces termes anéantit toute tentative de motivation et empêche l'apprenant de proposer ces termes lors de la construction d'hypothèses. Cette manière de formuler ne constitue nullement un moment didactique propice au déploiement d'un raisonnement scientifique de la part de l'apprenant. En conclusion, ce texte ne peut, en aucun cas, permettre la mise en œuvre d'un travail de problématisation nécessaire pour 
motiver et pour engager l'apprenant dans un raisonnement scientifique signifiant et générateur d'apprentissages en termes de savoirs et compétences visés par l'éducation relative à l'environnement.

\subsection{3 - Tableau de quelques dimensions de travail de problématisation}

Traits majeurs d'une activité de problématisation d'un phénomène, d'un concept ou d'un fait scientifique (selon le cadre théorique) du Texte introductif1 ou situation problématique proposé par le manuel scolaire :

\begin{tabular}{|c|c|c|c|c|c|}
\hline $\begin{array}{l}\text { Contribution à la } \\
\text { problématisation et } \\
\text { au questionnement } \\
\text { pour inciter à la } \\
\text { construction d'un } \\
\text { espace-problème }\end{array}$ & $\begin{array}{l}\text { Faits } \\
\text { empirique } \\
\text { s ou faits } \\
\text { établis } \\
\text { scientifiqu } \\
\text { ement }\end{array}$ & $\begin{array}{l}\text { Demande } \\
\text { explicite } \\
\text { de } \\
\text { productio } \\
\text { n } \\
\text { d'hypoth } \\
\text { èses }\end{array}$ & $\begin{array}{l}\text { Modélisati } \\
\text { on } \\
\text { (demande } \\
\text { explicite } \\
\text { de } \\
\text { modélisati } \\
\text { on) }\end{array}$ & $\begin{array}{l}\text { Dimension } \\
\text { langagière dans le } \\
\text { texte ou les } \\
\text { questions } \\
\text { proposées }\end{array}$ & $\begin{array}{l}\text { Lien évoqué } \\
\text { ou non par le } \\
\text { texte ou les } \\
\text { questions } \\
\text { entre } \\
\text { problème et } \\
\text { savoirs } \\
\text { scolaires }\end{array}$ \\
\hline 0 & 0 & + & 0 & 0 & 0 \\
\hline
\end{tabular}

4.2 - Situation 2 : (livre: Sciences de la vie et de la terre Tronc Commun scientifique ; Chapitre 5 : Activité 2 : la protection des équilibres naturels ; $p$ : 102 ; manuel Al Moussaid SVT Collection).

\subsection{1 -Texte introductif de l'activité 2 du chapitre 5 du manuel}

Le rythme élevé de la dégradation et les déséquilibres des milieux naturels nécessitent l'intervention de l'homme afin de protéger les écosystèmes, et d'assurer une bonne gestion de l'exploitation des ressources naturelles.

Quelles sont les procédures utilisées pour protéger l'environnement?

\subsection{2- Analyse critique du texte introductif}

(Les auteurs de ce manuel proposent ce texte comme situation problématique permettant l'introduction de cette thématique).

A la lecture de texte introductif, les remarques suivantes sont relevées

- Le texte proposé évoque des termes qui ne devraient pas être utilisés et divulgués car ils doivent faire l'objet d'une réflexion et un effort collectif, et ce,en organisant des activités à l'issue desquelles les apprenants proposent ces termes sous formes d'hypothèses. Le fait de révéler ces termes rend impossible toute activité visant la motivation et l'initiation au raisonnement scientifique ;

- Le choix du terme de procédure n'est pas fondé et ne serait pas pertinent et adéquat pour introduire l'idée de protection de l'environnement ; 
- Le choix des termes et des concepts ne seraient pas suffisamment fondés et les auteurs ne semblent pas conscients du sens scientifique de certains termes et concepts utilisés dans le texte. En conséquence, cette multitude de concepts utilisés empêche toute démarche de compréhension ou de problématisation ;

- Le texte ressemble plus à un résumé, mal articulé, d'une leçon et ne peut en aucun cas servir de support pour aider à la problématisation ;

- Le texte en question propose des conclusions et des idées qui doivent être proposées non pas par les auteurs mais par les apprenants dans une démarche d'initiation à l'investigation et à la démarche scientifique ;

- Une lecture de ce texte ne permettrait pas de relever des faits empiriques ou des faits établis scientifiquement, nécessaires pour permettre à l'apprenant de s'initier à la démarche scientifique en proposant des hypothèses et en imaginant des procédés permettant de tester ces hypothèses ;

- Les termes utilisés, la structuration et les éléments fournis par le texte ne permettent, en aucun cas, une utilisation didactique de ce texte pour servir de situation problématique, d'une part, une utilisation de ce texte pour aider à la problématisation, d'autre part. La logique d'élaboration du texte ainsi queles idées et faits proposés par le texte ne permettraient pas d'engager l'apprenant dans une démarche de production d'hypothèse ;

Par conséquent, le texte ne pourrait être utilisé comme texte didactique visant la mise en œuvre d'une démarche de problématisation et par voie de conséquence d'initiation aux raisonnements scientifiques dans une perspective scolaire.

\subsection{3- Tableau de quelques dimensions de travail de problématisation}

\begin{tabular}{|l|l|l|l|l|l|}
\hline $\begin{array}{l}\text { Contribution à la } \\
\text { problématisation } \\
\text { et au } \\
\text { questionnement } \\
\text { pour inciter à la } \\
\text { construction } \\
\text { d'un espace- } \\
\text { problème }\end{array}$ & $\begin{array}{l}\text { Faits empiriques } \\
\text { ou faits établis } \\
\text { scientifiquement }\end{array}$ & $\begin{array}{l}\text { Demande } \\
\text { explicite de } \\
\text { production } \\
\text { d'hypothèses }\end{array}$ & $\begin{array}{l}\text { Modélisation } \\
\text { (demande } \\
\text { explicite de } \\
\text { modélisation) }\end{array}$ & $\begin{array}{l}\text { Dimension } \\
\text { langagière } \\
\text { dans le } \\
\text { texte ou } \\
\text { les } \\
\text { questions } \\
\text { proposées }\end{array}$ & $\begin{array}{l}\text { Lien } \\
\text { évoqué ou } \\
\text { non par le } \\
\text { texte ou } \\
\text { les } \\
\text { questions } \\
\text { entre } \\
\text { problème } \\
\text { et savoirs } \\
\text { scolaires }\end{array}$ \\
\hline 0 & & & 0 & 0 & 0 \\
\hline
\end{tabular}


Traits majeurs d'une activité de problématisation d'un phénomène, d'un concept ou d'un fait scientifique (selon le cadre théorique) Texte introductif 2ou situation problématique proposé par le manuel scolaire

4.3 - Situation 3 :(livre : Sciences de la vie et de la terre Tronc Commun scientifique; Chapitre II : Les équilibres naturels et quelques aspects de leur déséquilibre, $p$ : 90, Editions : IMARSI).

\subsection{1 -Texte introductif du chapitre 2}

Le Maroc se caractérise par la diversité de son climat, ses reliefs, son sol, ses écosystèmes et l'abondance de ses ressources naturelles agricoles, minières et halieutiques.

Son régime économique dépend surtout de ressources terrestres et maritimes qui ne cessent de se dégrader avec l'augmentation des besoins de ses habitants.

$\checkmark$ Quelles sont les conséquences d'une exploitation non raisonnée de nos ressources naturelles ?

$\checkmark$ Quelles sont les aspects des déséquilibres naturels ? Et quelle relation avec les activités humaines ?

$\checkmark \quad$ Ouel est le rôle humain dans la protection de la nature ?

\subsection{2- Analyse critique de la situation 3}

Suite à une première lecture de ce texte, pour introduire le concept d'équilibre naturel, les remarques suivantes sont à relever :

- Le texte véhicule des propositions qui renvoient à plusieurs problématiques et thématiques et ne permettraient nullement à l'apprenant de se focaliser et de tenir une conduite d'investigation pour discerner des faits qui puissent aider à construire des hypothèses relativement à des causes ou des actions réparatrices en rapport avec une problématique écologique ou environnementale ;

- Un nombre important de termes et de concepts rend difficile la compréhension et le développement d'un raisonnement scientifique pour s'acheminer vers la construction de la problématique auquel renvoie le chapitre II ;

- Le texte introductif ne propose pas de faits empiriques, de constats, de données ou de faits scientifiques établis qui puissent suggérer des hypothèses sur les conséquences d'une exploitation abusive des ressources naturelles et permettre ainsi de développer une problématisation ;

- La structuration du texte ne serait pas pertinente pour aider l'apprenant à développer un raisonnement scientifique, pour réaliser une problématisation de la thématique des équilibres naturels dans laquelle l'apprenant est invité à produire des hypothèses à partir de faits empiriques ou de faits établis scientifiquement; 
- En ce qui les questions proposées par les auteurs, l'absence d'une question importante sur les conditions, les causes et les pratiques courantes en relation avec l'exploitation abusives des ressources des écosystèmes est bien visible ;

- Parmi les questions formulées, aucune ne porte sur l'élaboration d'un texte et le travail langagier nécessaire dans toute démarche de problématisation et qui constituerait un exercice et une initiation à la démarche scientifique ;

En conséquence, le texte aurait peu d'intérêt didactique pour servir de contenu visant à problématiser les thématiques de déséquilibre des écosystèmes, de protection et de préservation des systèmes naturels.

\subsection{3- Tableau de quelques dimensions de travail de problématisation Traits majeurs d'une activité de problématisation d'un phénomène, d'un concept ou d'un fait scientifique (selon le cadre théorique) Texte introductif3 ou situation problématique proposé par le manuel scolaire}

\begin{tabular}{|l|l|l|l|l|l|}
\hline $\begin{array}{l}\text { Contribution à la } \\
\text { problématisation et } \\
\text { au questionnement } \\
\text { pour inciter à la } \\
\text { construction d'un } \\
\text { espace-problème }\end{array}$ & $\begin{array}{l}\text { Faits empiriques } \\
\text { ou faits établis } \\
\text { scientifiquement }\end{array}$ & $\begin{array}{l}\text { Demande } \\
\text { explicite de } \\
\text { production } \\
\text { d'hypothèses }\end{array}$ & $\begin{array}{l}\text { Modélisation } \\
\text { (demande } \\
\text { explicite de } \\
\text { modélisation) }\end{array}$ & $\begin{array}{l}\text { Dimension } \\
\text { langagière } \\
\text { dans le } \\
\text { texte ou } \\
\text { les } \\
\text { questions } \\
\text { proposées }\end{array}$ & $\begin{array}{l}\text { Lien évoqué } \\
\text { ou non par le } \\
\text { texte ou les } \\
\text { questions } \\
\text { entre } \\
\text { problème et } \\
\text { savoirs } \\
\text { scolaires }\end{array}$ \\
\hline 0 & 0 & & 0 & 0 \\
\hline
\end{tabular}

\section{Discussion}

Après une analyse des textes proposés, il s'est avéré qu'ils présentent les mêmes caractéristiques que ce soit sur le plan de la structuration ou du contenu en termes d'idées, de faits ou de formulation. Ceci rend possible le développement d'une interprétation globale, commune et qui serait valable pour l'ensemble des textes introductifs proposés par les différents manuels retenus dans le présent travail.

Les textes introductifs ou les situations problématiques des différents manuels seraient formulées sous forme de textes- synthèses, de résumés qui mettent en avant des vérités scientifiques scolaires. Ces textes ne pourraient, en aucun cas, constituer des contenus qui aideraient à problématiser des phénomènes, des thématiques ou des concepts à l'étude. En effet, ils ne comportent pas les faits empiriques ou scientifiques nécessaires à tout travail de problématisation. Il semblerait que les auteurs des manuels ne possèdent pas une culture épistémologique nécessaire à l'élaboration d'un texte 
introductif pertinent en relation avec l'objectif de problématisation. Une ignorance des aspects épistémologiques seraient à l'origine de l'incapacité de saisir ce qu'est une situation problématique et comment formuler une situation qui aurait des implications didactiques possibles. L'analyse de ces situations révèle une confusion totale sur le sens et les traits majeurs d'une situation problématique. Les auteurs des manuels semblent se préoccuper davantage sur la formulation des questions qui ne sont pas toujours articulées à un contenu ; ces formulations présentent un mélange d'idées, de données et de faits qui ne permettraient pas toujours de dégager le problème visé. Ainsi, toute activité de problématisation serait difficile voire impossible à mettre en place. En outre, le texte proposé renvoiegénéralementplusieurs problèmes en relation avec une multitude d'idées et de notions qui empêchent l'apprenant de se focaliser sur une problématique particulière.

En ce qui concerne les implications didactiques de ces situations, il semblerait que l'accent a été mis sur l'utilisation des situations problématiques comme des moments didactiques servant à créer la motivation. Elles ne seraient pas exploitées pour le développement de la leçon et la conceptualisation. Les auteurs de ces manuels ne semblent pas conscients du lien étroit et important entre problématisation et conceptualisation, d'une part, entre problèmes et savoirs scientifiques, d'autre part. Ces auteurs auraient ainsi une conception très étroite de l'idée de problématisation. Une conception qui ne permettrait pas de concevoir, d'élaborer et de mettre en œuvre une activité de problématisation visant la conceptualisation de la thématique ou du concept à l'étude.

Relativement à la relation singulière entre problème et solution, les textes proposés ne permettraient pas de mettre en valeur cette relation. Les textes proposés ne permettent pas de déduire et de percevoir cette relation particulière entre problème et solution. Il y a lieu de souligner que cette relation renvoie en fait aux hypothèses possibles et à la nécessitéd'une explication qui correspond en fait à l'hypothèse plausible.

Relativement à la dimension langagière de l'activité de problématisation, les textes proposés ne comportent pas de questions ou d'idées qui incitent explicitement à un travail langagier ou de mise en texte nécessaire dans toute activité de problématisation. Il semblerait que les auteurs de ces situations ignorent le caractère langagier de tout travail de problématisation. En effet, les situations proposées ne comportent pas de questions ou d'invitations à la mise en texte ou la mise en récit d'un processus de problématisation visant un fait, un phénomène ou une problématique. Il s'avère alors que la culture épistémologique des auteurs est atrophiée voire absente. En revanche, cette culture est nécessaire pour développer des compétences qui permettraient de concevoir, d'élaborer et de mettre en œuvre des situations problématiques visant la motivation et le traitement didactique 
d'un phénomène ou d'une thématique visant de réaliser les conditions de possibilité d'une conceptualisation possible par l'apprenant.

\section{Conclusion}

$\mathrm{Au}$ terme de cette étude, l'accent est à mettre sur quelques résultats relatifs aux analyses critiques des textes introductifs qui constituent en fait des situations problématiques proposées par des manuels scolaires en rapport avec des thématiques écologiques et environnementales. L'analyse a été menée à travers des grilles d'analyse et un cadre théorique suffisamment fondé pour servir de fond théorique à partir duquel une nouvelle démarche d'analyse est à mettre en place. Toutes les analyses menées avaient pour but d'évaluer les potentialités des textes proposés en termes de problématisation et ce en rapport avec la place et l'importance de ce processus de problématisation dans toute démarche visant l'accès au savoir scientifique et son appropriation. Ce travail présente un enjeu théorique, dans la mesure où il participe aux recherches sur les processus de problématisation. La méthodologique adoptée a été inspirée du cadre théorique développé par le CREN. S'il faut retenir une spécificité de la problématisation scientifique, c'est son fonctionnement dans deux trajets opposés : vers la solution et vers la reconstruction du problème.

Les textes analysés laissent entrevoir un potentiel didactique mince eu égard à toute activité de problématisation qui serait un préalable pour amorcer une leçon visant l'enseignement des sciences. Les textes proposés sont loin de constituer des supports didactiques permettant de mettre en avant des données empiriques et des nécessités accessibles aux apprenants et qui se prêtent à une éventuelle exploitation didactique. De même, ces textes ne fournissent ni les données ni la structure, ni les questions qui inciteraient à engager un travail langagier ou de mise en texte nécessaire dans toute activité de problématisation en sciences.

En conséquence, force est de constater que l'élaboration d'un texte, pouvant servir de situation problématique de départ, n’est ni simple ni évidente et requiert nombre de compétences académiques, épistémologiques et didactiques. Il apparait que les auteurs des manuels ne seraient pas détenteurs de ces compétences ; ceci expliquerait peut-être l'intérêt didactique très limité de ce texte.

La conception d'un texte introductif impliquerait une réflexion et un travail en profondeur par une équipe de spécialistes susceptible de concevoir des textes qui puissent servir de document introductif et support d'une activité didactique menée par l'apprenant et dans laquelle ce dernier est engagé mentalement dans un processus d'investigation et de démarche scientifique. 


\section{References:}

1. Aikenhead, G. S. (2003, August). Review of research on humanistic perspectives in science curricula. In 4th Conference of the European Science Education Research Association (ESERA), Research and the Quality of Science Education. Noordwijkerhout, The Netherlands (august 19-23).

2. Bachelard, G. (1986). La formation de l'esprit scientifique. Paris : Vrin, (1re édition 1938)

3. Bachelard, G. (1972). L'engagement rationaliste. Paris : Les presses Universitaires de France

4. Bardin, L. (1977). L'analyse de contenu. Paris : PUF.

5. Berlin, J.P. (2002). Une cerise sur le gâteau OGM. Le Monde, 4 novembre

6. Brunet, P. (1998). Enseigner et apprendre par problèmes scientifiques dans les sciences de la vie. État de la question. Aster, 27, 145-181.

7. Canguilhem, G. (1988). Idéologie et rationalité dans l'histoire des sciences de la vie. Paris: Vrin.

8. Dewey, J. (1933). How we think: a restatement of the relation of reflective thinkingto the education process. Chicago, Ill: H. Regnery.

9. Dewey, J. (1930). Experience and education. Paris : Armand Colin

10. Fabre, M. \& Musquer, A. (2009). Comment aider l'élève à problématiser ? Les inducteurs de problématisation. Revue les Sciences de l'éducation- Pour l'Ère nouvelle, L'autorité à l'heure de la démocratie, vol. 42, n³, pp. 111-129. ISSN 0755-9593. ISBN 978-2918337-01-0

11. Fabre, M. (2005). Formation et problématisation. Recherche et Formation, (b) $\mathrm{n}^{\circ} 48$.

12. Fabre, M. (2005). Deux sources de l'épistémologie des problèmes: Dewey et Bachelard. Les Sciences de l'éducation-Pour l'Ère nouvelle, 38(3), 53-67

13. Fabre, M. \& Orange, C. (1997). Construction des problèmes et franchissements d'obstacles. Aster, 24, 37-57.

14. Fabre, M.(1993).Statut et fonction du problème dans l'enseignement des sciences.Les Sciences de l'éducation, Pour l'ère nouvelle, 26 (4/5).

15. Fabre, M. (1999). Situations-problèmes et savoir scolaire. Paris: Presses universitaires de France

16. Kolsto, S.D. (2001). « To trust or not to trust, ... »-pupils’ ways of judging information encountered in a socio-scientific issue. International Journal of Science Education 23, 877-901.

17. Kuhn, D.(2001).How do people know ?,Psychological Science 12,1-8.

18. Lage, B. (2007). Enseignement par compétences et changement de paradigmes étude des représentations des enseignants à propos de leur 
métier, de l'apprentissage et de l'enseignement. Université de Rouen, UFR psychologie sociale et sciences de l'éducation.

19. Melhaoui, M., El Hafid, L., Amamou, B., \& Hammouti, A. (2004). L'échec à l'université Marocaine : bilan des principales causes et attentes de la pédagogie universitaire. Dans AIPU. Actes du 21eCongrès international de l'AIPU du 3 au 7 mai. Publication de l'université Cadi Ayyad, Marrakech, Maroc, p. 22-23.

20. Orange, C. (2002). Apprentissages scientifiques et problématisation. Les Sciences de l'éducation, pour l'ère nouvelle. 35, 1, 25-42.

21. Orange, C. (2005). Problème et problématisation. ASTER, ( $a), n^{\circ} 40$.

22. Orange, C. (2005). Problématisation et conceptualisation en sciences et dans les apprentissages scientifiques. Les sciences de l'éducation Pour l'Ère nouvelle, (b), vol.38, $\mathrm{n}^{\circ} 3$

23. Orange, C. (2005). Problématisation et conceptualisation en sciences et dans les apprentissages scientifiques. Les Sciences de l'éducation Pour l'Ère nouvelle, La problématisation : approches épistémologiques, vol. 38, n 3, pp. 69-94. ISSN 0755-9593. ISBN 29506879-4-6.

24. Orange, C. (2019). Problématisation en sciences et dans les apprentissages scientifiques. [En ligne],(page consultée le 30 juillet2019).Disponible sur : http://acireph.org/IMG/pdf/cp11/cp11_art4_Orange.pdf

25. Orange, C. (2019). Quel Milieu pour l'apprentissage par problématisation en sciences de la vie et de la terre ? Education et didactique [En ligne], vol 1 - $\mathrm{n}^{\circ} 2 \mid$ septembre 2007, mis en ligne le 01 septembre 2009, (page consultée le 30 avril.Disponible sur: URL:http://journalsopenedition.org/educationdidactique/

26. Pauen, S. \& Wilkzning, F. (1997). Children's Analogical Reasoning about NaturalPhenomena. Journal of Experimental Child Psychology 67, 90-113.

27. Poirier Proulx, L. (1999). La résolution de problèmes en enseignement. Paris :Bruxelles ; De Boeck.

28. Popper, K. (1972). La connaissance objective. Paris : Aubier

29. Popper, K. (1984). La logique de la découverte scientifique. Paris : Payot, (1re édition 1959).

30. Popper, K. (1985). Conjecture et réfutations. Paris : Payot

31. Popper, K. (1963). Conjectures et réfutations. Paris : Payot, 1985 (1re édition)

32. Rapport interne (2015). Ministère de l'enseignement scolaire. Document interne sur la réforme de l'enseignement des SVT tronc commun et cycle qualifiant 
33. Sadler, T.D., Chambers, W., \& Zeidler , D. (2004). Student conceptualizations of the nature of science in response to a socioscientificissue. International Journal of Science Education26, 387-409.

34. Simonnex, L. (2001). Role-play or debate to promote students' argumentation and justification on an issue in animal transgenesis. International Journal of Science Education, 23, 903-927

35. Simonnex, L. (2002). Analysis of classroom debating strategies in the field of biology. Journal of Biological Education 37, 9-12

36. Tbilissi, M. (1977). Conférence intergouvernementale sur l'éducation relative à l'environnement, Tbilissi, URSS, 14-26 octobre: rapport final

37. UNESCO (2009). UNESCO Word Conference on Education for Sustainable Development.31 mars-2 avril, Bonn. En ligne : $<$ http://www.esd-world-conference-2009.org/en/whatsnew/news-detail/item/conference-proceedings-published.html>.

38. Zimmerman, C. (2000). The Development of Scientific Reasoning Skills. DevelopmentalReview 20, 99-149 